\title{
Effects of Local Item Dependence on the Fit and Equating Performance of the Three-Parameter Logistic Model
}

\author{
Wendy M. Yen \\ CTB/McGraw-Hill
}

\begin{abstract}
Unidimensional item response theory (IRT) has become widely used in the analysis and equating of educational achievement tests. If an IRT model is true, item responses must be locally independent when the trait is held constant. This paper presents several measures of local dependence that are used in conjunction with the three-parameter logistic model in the analysis of unidimensional and two-dimensional simulated data and in the analysis of three mathematics achievement tests at Grades 3 and 6 . The measures of local dependence (called $Q_{2}$ and $Q_{3}$ ) were useful for identifying subsets of items that were influenced by the same factors (simulated data) or that had similar content (real data). Item pairs with high $Q_{2}$ or $Q_{3}$ values tended to have similar item parameters, but most items with similar item parameters did not have high $Q_{2}$ or $Q_{3}$ values. Sets of locally dependent items tended to be difficult and discriminating if the items involved an accumulation of the skills involved in the easier items in the rest of the test. Locally dependent items that were independent of the other items in the test did not have unusually high or low difficulties or discriminations. Substantial unsystematic errors of equating were found from the equating of tests involving collections of different dimensions, but substantial systematic errors of equating were only found when the two tests measured quite different dimensions that were presumably taught sequentially.
\end{abstract}

APPLIED PSYCHOLOGICAL MEASUREMENT

Vol. 8, No. 2, Spring 1984, pp. 125-145

(C) Copyright 1984 Applied Psychological Measurement Inc. 0146-6216/84/020125-21\$2.30
The fundamental assumption of the three-parameter logistic model is that

$$
\begin{aligned}
& P_{i}\left(\theta_{k}\right)= \\
& c_{i}+\frac{1-c_{i}}{1+\exp \left[-1.7 a_{i}\left(\theta_{k}-b_{i}\right)\right]},
\end{aligned}
$$

where $P_{i}\left(\theta_{k}\right)$ is the probability that the $k$ th examinee passes the $i$ th item,

$\theta_{k}$ is the trait value for the $k$ th examinee, and

$a_{i}, b_{i}$, and $c_{i}$ are the discrimination, difficulty, and lower asymptote for the $i$ th item.

Local item independence follows from Equation 1; if the model is true, the probability of correctly answering both items $i$ and $j$ is

$P_{i j}\left(\theta_{k}\right)=P_{i}\left(\theta_{k}\right) P_{j}\left(\theta_{k}\right)$.

Equation 2 defines pairwise local independence. If the model is true, then all higher order extensions of Equation 2 will hold as well.

In examining the fit of the model it is common to compare model predictions (Equation 1) with observations. This comparison traditionally has been between observed and predicted item characteristic curves, and there has been little direct investigation of local independence. There are many factors that can affect the fit of a model. One of the most obvious is multidimensionality. For many tests there is an a priori suspicion that test performance is not unidimensional and that a unidimensional model is not appropriate. This concern is particularly strong 
for achievement tests where several different types of content are tested (Traub, 1983). Such multidimensionality would be expected to have a particularly strong effect on the local independence of at least some of the items. Given this concern, it is desired to measure local dependence and examine its impact on the use of a unidimensional model-the three-parameter logistic model.

In this paper several measures of local dependence are proposed, and they are applied to simulated data and to data from six mathematics achievement tests. The effects of local dependence on a traditional measure of model fit are examined. Local dependence is also related to item parameter values and to the quality of test equatings.

\section{Method}

\section{Fit Measures}

$\mathbf{Q}_{1}$. Traditional measures of fit in item response theory (IRT) typically involve comparisons between observed and predicted item characteristic curves using a chi-square test such as $Q_{1}$ (Yen, 1981):

$Q_{1 i}=\sum_{r=1}^{10} \frac{N_{r}\left(O_{i r}-E_{i r}\right)^{2}}{E_{i r}\left(1-E_{i r}\right)}$.

$Q_{1 i}$ is the fit of the $i$ th item, and it is distributed approximately as a chi-square variable with seven degrees of freedom when the three-parameter model is true. To obtain $Q_{1}$ examinees are rank ordered on the basis of their trait estimates and then divided into deciles (10 cells with approximately equal numbers of examinees per cell). $N_{r}$ is the number of examinees in cell $r, O_{i r}$ is the observed proportion of examinees in cell $r$ that correctly answers item $i$, and $E_{a r}$ is the predicted proportion of examinees in cell $r$ that correctly answers item $i$. The predictions are obtained by using trait and item parameter estimates in Equation 1 and summing over examinees in cell $r$ :

$E_{i r}=\frac{1}{N_{r}} \sum_{k \varepsilon r}^{N_{r}} \hat{P}_{i}\left(\hat{\theta}_{k}\right)$

The fit of the entire test is $Q_{1}=\Sigma_{i=1}^{\prime} Q_{1 i}$, with $I$ items in the test.

$\mathbf{Q}_{2}$. Van den Wollenberg (1982) proposed a fit measure for the Rasch model, called $Q_{2}$, that he found is sensitive to multidimensionality. To obtain $Q_{2}$, a contingency table is created for each pair of items for each (overall test) score group (cell) as in Table 1. In this table $N_{r}$ is the number of examinees in cell $r, N_{i j}$ is the number of examinees in the cell that correctly answered both items $i$ and $j, N_{i r}$ is the number of examinees that correctly answered item $i, N_{i j r}$ is the number of examinees that correctly answered item $i$ and incorrectly answered item $j$, and so on.

Van den Wollenberg's fit statistic for the $r$ th cell is

$$
\begin{aligned}
Q_{2 i j r} & =\frac{\left[N_{i j r}-\mathrm{E}\left(N_{i j r}\right)\right]^{2}}{\mathrm{E}\left(N_{i j r}\right)} \\
& +\frac{\left[N_{i j r}-\mathrm{E}\left(N_{i j r}\right)\right]^{2}}{\mathrm{E}\left(N_{i j r}\right)} \\
& +\frac{\left[N_{i j r}-\mathrm{E}\left(N_{i j r}\right)\right]^{2}}{\mathrm{E}\left(N_{i j r}\right)} \\
& +\frac{\left[N_{i j r}-\mathrm{E}\left(N_{i j r}\right)\right]^{2}}{\mathrm{E}\left(N_{i j r}\right)} .
\end{aligned}
$$

The overall fit measure for item pair $i, j$ is the sum of the $Q_{2 i j r}$ values over the $R$ cells. Van den Wollenberg obtained the expected values for $Q_{2}$ using a procedure specifically associated with the Rasch model.

In generalizing $Q_{2}$ for the three-parameter model, 10 cells were used as defined for $Q_{1}$. The expectations were defined as

$$
\begin{aligned}
& \mathrm{E}\left(N_{i j r}\right)=N_{i r} N_{j r} / N_{r}, \\
& \mathrm{E}\left(N_{i j r}\right)=N_{i r} N_{j r} / N_{r}, \\
& \mathrm{E}\left(N_{i j r}\right)=N_{i r} N_{j r} / N_{r},
\end{aligned}
$$

and

$$
\mathrm{E}\left(N_{i j r}\right)=N_{i r} N_{j r} / N_{r} .
$$

This characterization of $Q_{2}$ appears consistent with Lord's (1953, p. 545) suggested procedure for examining local independence.

$Q_{2}$ takes the form of a Pearson chi-square statistic (Hays, 1973). There are 10 independent cells (observations) with one degree of freedom per cell, leading to the conclusion that $Q_{2}$ should follow a chi-square distribution with 10 degrees of freedom 
Table 1

Number of Examinees in Cell $r$ With Each Pattern of Item Scores

\begin{tabular}{cccc}
\hline \hline Item $i$ & \multicolumn{3}{c}{ Item $j$ Score } \\
\cline { 2 - 4 } Score & 0 & 1 & Total \\
\hline 0 & $N_{\bar{i} \bar{j} r}$ & $N_{\bar{i} j r}$ & $N_{\overline{i r}}$ \\
1 & $N_{i \bar{j} r}$ & $N_{i j r}$ & $N_{i r}$ \\
Total & $N_{\bar{j} r}$ & $N_{j r}$ & $N_{r}$ \\
\hline
\end{tabular}

when the null hypothesis (local independence) is true. The determination of the cell boundaries is dependent on the observed distribution of estimated trait values, which may affect the distribution of $Q_{2}$ and its degrees of freedom. The null distribution of $Q_{2}$ will be examined with simulation data.

Using the expectations in Equation 6, $Q_{2 i j r}$ $=N_{r} \phi_{i j r}^{2}$, where $\phi_{i j r}$ is the phi coefficient (correlation) between item scores in cell $r$. Binary scores (such as those for items) are pairwise independent if and only if they are uncorrelated, because the covariance of items $i$ and $j$ is $P_{i j}-P_{i} P_{j}$. Pairwise independence does not preclude higher order dependencies, though in most testing applications it is difficult to imagine important higher-order dependencies that are not reflected in pairwise dependence.

Although $Q_{2}$ offers a method of determining where local dependence exists, it does not reflect the positive or negative direction of the local dependence. It is easy to imagine positive local dependence when two or more items measure special traits that do not appear in the remainder of the test. Negative local dependence can appear between two sets of items that measure different traits. For example, imagine that the trait estimated by the unidimensional model is the average of two underlying traits and that to look for local dependence examinees are sorted into cells on the basis of this average. An examinee can be placed in a cell for moderate scorers by having moderate scores on both underlying traits, by having a low score on Trait 1 and a high score on Trait 2 , by having a high score on
Trait 1 and a low score on Trait 2, and so forth. This procedure will reveal a negative relationship between performance on items measuring Trait 1 and items measuring Trait 2 .

To estimate the direction of the relationship between items $i$ and $j, \sum_{r=1}^{10} \phi_{i j r}$ was obtained. The sign of this sum was then applied to $Q_{2}$ to create "signed $Q_{2}$."

$\mathbf{Q}_{3}$. The disadvantage of $Q_{2}$ and signed $Q_{2}$ is that the number and definition of the cells are arbitrary. Kingston and Dorans (1982) examined local dependence using a statistic that does not require cells - the correlation of item scores with the trait estimate partialled out $\left(r_{i j} \cdot \hat{\theta}\right)$. The disadvantage of this statistic is that it removes only the linear relationship between item scores and traits when it is known that items and traits have a nonlinear, logistic relationship. An alternative to $r_{i j \cdot \hat{\theta}}$ was examined that removed the nonlinear effects of $\hat{\theta}$ from the item scores. Define

$d_{i k}=u_{i k}-\hat{P}_{i}\left(\hat{\theta}_{k}\right)$,

where $u_{i k}$ is the score of the $k$ th examinee on the $i$ th item. Then, the correlation (taken over examinees) of these scores is

$Q_{3 i j}=r_{d^{\prime} d_{j}}$.

Because $d_{i k}$ and $d_{j k}$ are random error scores when the three-parameter model is true, they may be distributed approximately as bivariate normal variables with a zero correlation. Thus, when the model is true, Fisher's $r$-to- $z$ transformation of $Q_{3}$ may be distributed as a normal variable with mean equal to zero and variance equal to $1 /(N-3)$, where there are $N$ examinees involved in the calculation of the correlation. Kingston and Dorans (1982) noted that because item scores are involved in the calculation of $\hat{\theta}, r_{i j} \cdot \hat{\theta}$ values will tend to be slightly negative due to part-whole contamination. A similar effect may occur with $Q_{3}$. The distribution of the $z$ transformation of $Q_{3}$ will be examined with simulated data.

\section{Parameter Estimation}

LOGIST 5 Version 1.0 (Wingersky, Barton, \& Lord, 1982) was used to estimate item parameters and trait values for the three-parameter logistic 
model. The default options were used in running LOGIST. In estimating item parameters, omits were treated as incorrect answers and not-reached items were treated as "not reaches."

$Q_{2}$, signed $Q_{2}$, and $Q_{3}$ were obtained for each pair of items in each LOGIST run. Also, the average of the $Q_{1}$ values for each pair of items was obtained and called $\bar{Q}_{1}$. Examinees who did not reach an item were not included in the calculation of these statistics for that item.

\section{Simulated Data}

Tests and examinees. To examine the null distribution of $Q_{2}$ and the $z$ transformation of $Q_{3}$, simulated data were used. A simulated unidimensional test was created by using the parameters of the first 20 items of the Level F Reading Vocabulary test from the Comprehensive Tests of Basic Skills, Form U (CTBS/U; CTB/McGraw-Hill, 1981). These parameters were obtained from that test's standardization (CTB/McGraw-Hill, 1982a; Yen, 1983b). An additional unidimensional simulated test was created using the parameters of the first 40 items of that Reading Vocabulary test. Item responses for these two tests were generated from the three-parameter model (Equation 1) for 1000 simulees as described by Yen (in press).

Simulated data for three 30 -item multidimensional tests were also examined. Item responses for these tests were generated using a two-dimensional three-parameter logistic model whose general form is described by Reckase and McKinley (1983):

$$
\begin{aligned}
& P_{i}\left(\theta_{k 1}, \theta_{k 2}\right)= \\
& c_{i}+\frac{1-c_{i}}{1+\exp \left[-1.7 \sum_{s=1}^{2} a_{i s}\left(\theta_{k s}-b_{i s}\right)\right]}
\end{aligned}
$$

The parameters and traits in this model are the same as in Equation 1 with the addition of the subscript $s$, which specifies the two dimensions of the model.

The present study used item parameters from the "Easy" tests in "Configurations 1, 2, and 3" for the ' $\bar{b}_{2}-\bar{b}_{1}=0$ "' condition described by DoodyBogan and Yen (1983). Item responses generated for 2,000 "Low and Medium Ability" simulees in that study were used in the present study.
Table 2 displays the $a_{i s}$ values for the three multidimensional simulated conditions. These values indicate how the items were differentially affected by the two dimensions. In Configuration 1 , the correlation between $\theta_{1}$ and $\theta_{2}$ was approximately .60 , and the $a_{i s}$ and $b_{i s}$ values were chosen to have essentially zero correlations within and across dimensions. In Configuration 2 , the correlation between $\theta_{1}$ and $\theta_{2}$ was approximately .50 , and the $b_{i s}$ values were chosen to be essentially uncorrelated with each other and with the $a_{t s}$ values. In Configuration 3 , the correlation between $\theta_{1}$ and $\theta_{2}$ was approximately .50 , and the $b_{i s}$ values had a slightly negative correlation with the $a_{i s}$ values for the first dimension $(r=-.31)$ and a slightly positive correlation $(r=.09)$ for the second dimension. For every dimension and configuration, $\bar{b}=-.03$ and $S_{b}=.80$.

In running LOGIST for the simulated data the number of answer choices was set at four.

\section{Real Data}

Tests and examinees. The CTBS/U Mathematics Computation (MC) and Mathematics Concepts and Applications (MC\&A) tests were used. Level E (Grade 3) and Level G (Grade 6) were employed. The third- and sixth-grade students were tested as part of the Fall 1980 national standardization of CTBS/U. In addition to the CTBS/U tests, examinees took the Diagnostic Mathematics Inventory (DMI; Gessel, 1975a). DMI Level B was given at Grade 3 and Level $\mathrm{E}$ was given at Grade 6. The DMI items were classified as MC if they involved only adding, subtracting, multiplying, or dividing. All other DMI items were classified as MC\&A items. Table 3 contains the numbers of items and examinees.

The CTBS/U MC items have five answer choices and the CTBS/U MC\&A items have four answer choices. The DMI Level B items have five answer choices (except for one item with six answer choices), and the DMI Level $\mathrm{E}$ items have 8 (66\% of the items), $9(11 \%)$, or $10(23 \%)$ answer choices.

For each grade, the CTBS/U and DMI MC items were analyzed together by LOGIST, and the CTBS/ $\mathrm{U}$ and DMI MC\&A items were analyzed together. 
Table 2

Item Discriminations for Multidimensional Simulations

\begin{tabular}{|c|c|c|c|c|c|c|}
\hline \multirow[b]{3}{*}{ Item $(i$} & \multicolumn{6}{|c|}{ Configuration } \\
\hline & \multicolumn{2}{|c|}{1} & \multicolumn{2}{|c|}{2} & \multicolumn{2}{|c|}{3} \\
\hline & $a_{i l}$ & $a_{i 2}$ & $a_{i l}$ & $a_{i 2}$ & $a_{i l}$ & $\mathrm{a}_{\mathrm{i} 2}$ \\
\hline 1 & .8 & .6 & .6 & 1.8 & .5 & .6 \\
\hline 2 & 1.0 & .7 & .8 & 1.1 & .6 & .5 \\
\hline 3 & 1.1 & .6 & .9 & .9 & .6 & .6 \\
\hline 4 & 1.2 & .5 & 1.1 & .5 & .7 & .7 \\
\hline 5 & 1.4 & .9 & .7 & .0 & .7 & .7 \\
\hline 6 & 1.6 & .9 & .8 & .0 & .8 & .0 \\
\hline 7 & 1.8 & 1.1 & .8 & .0 & .8 & .0 \\
\hline 8 & 2.0 & .8 & .9 & .0 & .8 & .0 \\
\hline 9 & .5 & .9 & .9 & .0 & .8 & .0 \\
\hline 10 & .8 & 1.0 & 1.0 & .0 & .9 & .0 \\
\hline 11 & .8 & 1.1 & 1.0 & .0 & .9 & .0 \\
\hline 12 & .9 & 1.1 & 1.1 & .0 & .9 & .0 \\
\hline 13 & .7 & 1.2 & 1.1 & .0 & .9 & .0 \\
\hline 14 & 1.0 & 1.3 & 1.2 & .0 & 1.0 & .0 \\
\hline 15 & .6 & 1.4 & 1.2 & .0 & 1.0 & .0 \\
\hline 16 & 1.3 & 1.6 & 1.6 & .0 & 1.0 & .0 \\
\hline 17 & 1.2 & 1.8 & 2.0 & .0 & 1.0 & .0 \\
\hline 18 & 1.0 & 2.0 & .0 & .6 & 1.0 & .0 \\
\hline 19 & .6 & .7 & .0 & .7 & 1.1 & .0 \\
\hline 20 & .7 & .8 & .0 & .8 & 1.1 & .0 \\
\hline 21 & .8 & .8 & .0 & .8 & 1.1 & .0 \\
\hline 22 & .9 & .8 & .0 & .9 & 1.1 & .0 \\
\hline 23 & .9 & .9 & .0 & 1.0 & 1.2 & .0 \\
\hline 24 & .9 & 1.0 & .0 & 1.0 & 1.2 & .0 \\
\hline 25 & 1.0 & 1.0 & .0 & 1.0 & 1.2 & .0 \\
\hline 26 & 1.0 & 1.1 & .0 & 1.1 & 1.3 & .0 \\
\hline 27 & 1.1 & 1.0 & .0 & 1.2 & 1.4 & .0 \\
\hline 28 & 1.1 & 1.0 & .0 & 1.2 & 1.6 & .0 \\
\hline 29 & 1.1 & 1.2 & .0 & 1.3 & 1.8 & .0 \\
\hline 30 & 1.2 & 1.2 & .0 & 1.4 & 2.0 & .0 \\
\hline
\end{tabular}

Note. For explanatory purposes the item order has been changed from that in Doody-Bogan and Yen (1983).

In addition, the $\mathrm{MC}$ and $\mathrm{MC} \& \mathrm{~A}$ items were analyzed together. The number of answer choices for each test was set at the value described above; the number of answer choices was set at five for DMI Level B and at eight for DMI Level E.

Item configurations. Values of fit statistics were compared for groups of items using the KruskalWallis test (Siegel, 1956, pp. 184-185). Items were grouped into sets that appeared most likely to show local dependence, and the null hypothesis of the Kruskal-Wallis test was that the fit statistics within these sets arose from the same distribution as the fit statistics calculated across item pairs from different sets. The Kruskal-Wallis test comparing within-set and between-set fit statistics has a chisquare distribution with 1 degree of freedom under the null hypothesis.

Table 4 contains the definitions of the item configurations. Splitting the items by test (CTBS/U vs. DMI) is the first configuration for every grade-bycontent category. The CTBS, Forms $U$ and $V$, Test Coordinator's Handbook (CTB/McGraw-Hill, 
Table 3

Numbers of Items and Examinees

\begin{tabular}{ccccccc}
\hline & \multicolumn{2}{c}{ CTBS/U } & & \multicolumn{3}{c}{ DMI } \\
\cline { 2 - 3 } Grade & MC & MC\&A & & MC & MC\&A & N \\
\hline 3 & 36 & 40 & & 14 & 40 & 2184 \\
6 & 40 & 45 & & 48 & 61 & 2198 \\
\hline
\end{tabular}

1982b) and the DMI Teacher's Guide (Gessel, 1975b) were referred to for classifications of items into category objectives. For example, the Grade 3 $\mathrm{MC}$ test for CTBS/U has the following category objectives:

Add whole numbers,

Add decimals or fractions,

Subtract whole numbers,

Subtract decimals or fractions,

Multiply whole numbers,

Divide whole numbers.

The Grade $3 \mathrm{MC}$ test for DMI has the following category objectives:

Addition of whole numbers without regrouping (i.e., carrying),

Addition of whole numbers with regrouping,

Subtraction of whole numbers without regrouping

(i.e., borrowing),

Subtraction of whole numbers with regrouping,

Multiplication of whole numbers.

As can be seen in Table 4, various combinations and modifications of these category objectives were used to group items that appeared most likely to be locally dependent. For the MC\&A tests reconciliation of the category objectives for CTBS/U and DMI was more difficult than for the MC tests because different objective structures and terminology are used for these two tests, but sets of items most likely to be locally dependent were identified. Note that the same names were sometimes used for different item sets in different configurations (e.g., Sequences for Grade 3 MC\&A Data Sets 5 and 6).

In Data Sets 6 and 15 items were grouped that showed high $Q_{2}$ values. For example, in Data Set
6 the Graph Reading items were two CTBS/U items linked to the same graph, the Clock items all involved reading clocks, the Nearest Ten items involved rounding to the nearest 10 , and so forth. The Other items were all the remaining items that did not show high $Q_{2}$ values.

Trait estimation and comparison. Trait level estimates were obtained for different subsets of items using the item parameter estimates described above. In estimating trait levels, omits and not reaches were treated as wrong answers. Floors and ceilings for the trait estimates were obtained as described by Yen (1983b).

Grade 3 MC\&A (for both CTBS/U and DMI) and Grade $6 \mathrm{MC}$ (for both CTBS/U and DMI) were selected for the examination of trait estimates. For Grade 3 MC\&A, trait estimates based on CTBS/ $\mathrm{U}$ items were compared with trait estimates based on DMI items. Three additional trait estimates were obtained. The first was composed of the 27 locally dependent items shown in Data Set 6 in Table 4. The second trait estimate (Control 1) was composed of 26 items not included in the first trait estimate, and the third trait estimate (Control 2) was based on 25 items not included in the first or second trait estimate. Because the items in the locally dependent set tended to be adjacent pairs, the items chosen for Controls 1 and 2 also tended to be adjacent pairs.

For Grade $6 \mathrm{MC}$, trait estimates based on CTBS/ $\mathrm{U}$ items were compared with trait estimates based on DMI items. Three traits based on locally dependent items were also examined: items involving computations with whole numbers (36 items), items involving decimals ( 36 items), and items involving fractions (16 items). The computations involving different types of numbers tended to be interspersed 
Table 4

Item Configurations for Examining Local Dependence

\begin{tabular}{|c|c|c|c|c|}
\hline \multirow{2}{*}{$\begin{array}{l}\text { Data } \\
\text { Set }\end{array}$} & \multirow[b]{2}{*}{ Grade } & \multicolumn{2}{|c|}{ Test } & \multirow[b]{2}{*}{ Item Sets } \\
\hline & & $\overline{\mathrm{CTBS} / \overline{\mathrm{U}}}$ & $\overline{\mathrm{DMI}}$ & \\
\hline 1 & 3 & MC & MC & CTBS/U (36) ; DMI (14) \\
\hline 2 & 3 & MC & $\mathrm{MC}$ & $\begin{array}{l}\text { Add (16); Subtract (16); } \\
\text { Mult (12); Divide (6) }\end{array}$ \\
\hline 3 & 3 & MC & MC & $\begin{array}{l}\text { Add without regroup (7); Add } \\
\text { with regroup (9); Subtract } \\
\text { without regroup (9); Subtract } \\
\text { with regroup (7); Mult by } 5 \\
\text { (4);Other mult (8); Divide } \div \\
\text { (3);Divide } \sqrt{\text { (3) }}\end{array}$ \\
\hline 4 & 3 & $M C \& A$ & $M C \& A$ & CTBS/U (40);DMI (40) \\
\hline 5 & 3 & $\mathrm{MC} \& A$ & $\mathrm{MC} \& \mathrm{~A}$ & $\begin{array}{l}\text { Counting \& Matching (6) ; Numer- } \\
\text { ation (10); Number theory (10); } \\
\text { Measurement (17); Geometry (7); } \\
\text { Problem solving (10); Number } \\
\text { Sentences (17); Sequences (3) }\end{array}$ \\
\hline 6 & 3 & $M C \& A$ & $M C \& A$ & $\begin{array}{l}\text { Graph reading (2);Clock (3); } \\
\text { Nearest ten (2); Place value } \\
\text { (2);Number sentences (2); Even } \\
\text { numbers (2);Before (2); Number } \\
\text { line (2);Multiply (4); Fractions } \\
\text { (2);Sequences (2); Shapes (2); } \\
\text { Other (53) }\end{array}$ \\
\hline 7 & 3 & MC & $\mathrm{MC} \& \mathrm{~A}$ & CTBS/U $(36) ; \mathrm{DMI}(40)$ \\
\hline 8 & 3 & $M C \& A$ & $\mathrm{MC}$ & CTBS/U (40);DMI (14) \\
\hline 9 & 6 & MC & MC & CTBS/U (40); DMI (48) \\
\hline 10 & 6 & MC & MC & $\begin{array}{l}\text { Add (21); Subtract (22); Mult } \\
\text { (23); Divide (22) }\end{array}$ \\
\hline 11 & 6 & MC & $\mathrm{MC}$ & $\begin{array}{l}\text { Whole numbers (36); Decimals } \\
\text { (36); Fractions (16) }\end{array}$ \\
\hline 12 & 6 & $\mathrm{NC}$ & MC &  \\
\hline 13 & 6 & $\mathrm{MC} \& \mathrm{~A}$ & $M C \& A$ & CTBS/U (45);DMI (61) \\
\hline 14 & 6 & $\mathrm{MC} \& \mathrm{~A}$ & $\mathrm{MC} \& \mathrm{~A}$ & $\begin{array}{l}\text { Number sentences (18); Measure- } \\
\text { ment (18); Geometry (18); Problem } \\
\text { solving (19); Numeration (18); } \\
\text { Number theory (10); Sequences } \\
\text { (2); Inequalities, Odds,Multi- } \\
\text { ples (3) }\end{array}$ \\
\hline 15 & 6 & $\mathrm{MC} \& \mathrm{~A}$ & $M C \& A$ & $\begin{array}{l}\text { Greater than (2); Fractions (8); } \\
\text { Estimation (4); Sequences, } \\
\text { Inequalities, Odds (4);Conven- } \\
\text { tional measurement (2);Place } \\
\text { value DMI (2); Expanded nota- } \\
\text { tion (2);Place value CTBS(2); } \\
\text { Other (80) }\end{array}$ \\
\hline 16 & 6 & MC & $M C \& A$ & CTBS/U (40); DMI (61) \\
\hline 17 & 6 & $\mathrm{MC} \& \mathrm{~A}$ & $\mathrm{MC}$ & CTBS/U (45) ; DMI (48) \\
\hline
\end{tabular}

Note. The number of items in a set is in parentheses following the name of the set. 
in CTBS/U and grouped in DMI. Three additional traits were: Control 1 , a set of 36 items interspersed across both CTBS/U and DMI; Control 2, a set of 36 items not included in Control 1; and Control 3, a 16-item subset of the items in Control 2 .

Several statistics were used to compare different trait estimates: (1) the correlation, $r$; (2) standardized difference between means; (3) ratio of standard deviations; (4) standardized root mean squared difference (SRMSD); and (5) an estimate of local bias (Yen, 1983a). The standardized difference between means is the difference in mean scores for the two sets of traits divided by a pooled estimate of the standard deviation: $\bar{S}=\left[\left(S_{1}^{2}+S_{2}^{2}\right) / 2\right]^{1 / 2}$, where $S_{1}^{2}$ and $S_{2}^{2}$ are the variances of the two sets of scores. The standardized root mean squared difference is the square root of the mean squared difference between examinees' trait estimates, divided by $\bar{S}$.

To estimate local bias, examinees were rank ordered on the basis of their scores and grouped into quintiles (five cells with equal numbers of examinees in the cells). The average of the two scores being compared was used for the grouping. Within each cell the standardized difference between means was found. The average over cells of the absolute values of the' standardized mean differences was taken as the summary measure of local bias.

\section{Results}

\section{Simulations}

Unidimensional data. The mean and variance of $Q_{1}$ for the unidimensional simulation were 10.8 and 58.1 for the 20-item test and 7.1 and 12.5 for the 40 -item test. A goodness-of-fit test was conducted to determine if the $Q_{1}$ values had chi-square distributions with 7 degrees of freedom. In this test 10 cells were created with equal numbers of $Q_{1}$ values expected in each cell. The expectations were generated using a chi-square distribution with 7 degrees of freedom. The significance probabilities for these tests were .07 for the 20 -item simulation and .99 for the 40 -item simulation.

For the unidimensional simulation the mean and variance of the $Q_{2}$ values were 10.3 and 19.4 for the 20 -item test and 9.4 and 23.9 for the 40 -item test. A goodness-of-fit test was conducted to determine if the $Q_{2}$ values had chi-square distributions with 10 degrees of freedom. In this test 10 cells were created with equal numbers of $Q_{2}$ values expected in each cell. The expectations were generated using a chi-square distribution with 10 degrees of freedom. The significance probabilities for these tests were .36 for the 20 -item simulation and $<.001$ for the 40-item simulation. The $Q_{2}$ values for the 20 -item simulation looked like they had a chi-square distribution with 10 degrees of freedom, but the $Q_{2}$ values for the 40 -item simulation had more small values of $Q_{2}$ than expected for a chi-square distribution with 10 degrees of freedom. Thus, though the null hypothesis about the distribution of the $Q_{2}$ value was upheld only for the 20 -item test, the deviations from the expected distribution for the 40 -item test were not in the upper tail of the distribution where hypothesis testing is done. Large values of $Q_{2}$ that would be unlikely for a chi-square distribution with 10 degrees of freedom were unlikely in the simulated data.

The means of the $Q_{3}$ statistics were -.05 for the 20 -item test and -.02 for the 40 -item test. The significance probabilities of the chi-square tests that the $z$ transformations of these $Q_{3}$ values came from normal distributions with a mean of zero and a variance of $1 /(N-3)$ were $<.001$ for both the 20 and 40 -item tests. The correlations were more negative than expected by the null hypothesis.

Two-dimensional data. The means and variances of the $Q_{1}$ values for the two-dimensional simulations were 13.4 and 121.7 for Configuration 1, 9.7 and 20.0 for Configuration 2, and 8.0 and 15.8 for Configuration 3 . The significance probabilities of the tests that these $Q_{1}$ values came from a chi-square distribution with 7 degrees of freedom were $<.001$ for Configuration $1, .01$ for Configuration 2, and .86 for Configuration 3. For Configurations 1 and 2 there were more large $Q_{1}$ values than expected from the null distribution. The means and variances of the $Q_{2}$ values for the twodimensional simulated data were 10.1 and 34.6 for Configuration 1, 17.4 and 75.7 for Configuration 2, and 11.0 and 29.2 for Configuration 3. 
The significance probabilities of the tests that these $Q_{2}$ values came from a chi-square distribution with 10 degrees of freedom were .10 for Configuration $1,<.001$ for Configuration 2 , and .01 for Configuration 3. For Configurations 2 and 3 there were more large $Q_{2}$ values than expected from the null distribution. The mean $Q_{3}$ value was -.03 for each of the three configurations. The significance probabilities that the $z$ transformations of the $Q_{3}$ values came from normal distributions with zero means and variances of $1 /(N-3)$ were $<.001$ for each of the three configurations. For Configurations 1 and 3 there were more large negative $z$ values than expected, and for Configuration 2 there were more extreme negative and positive $z$ values than expected from the null distribution.

For Configurations 1,2 , and 3 the correlations between $\bar{Q}_{1}$ and $Q_{2}$ were $-.11,-.09$, and .00, the correlations between $\bar{Q}_{1}$ and $Q_{3}$ were -.05 , -.03 , and -.10 , and the correlations between signed $Q_{2}$ and $Q_{3}$ were .70, .94, and .75. While $\bar{Q}_{1}$ appeared to measure a different aspect of item fit than $Q_{2}$ and $Q_{3}$, these latter two statistics ap- peared to measure very similar properties of the items.

Items were grouped into sets that might be locally dependent, and the Kruskal-Wallis test was performed to see if the fit values within sets came from the same distribution as the fit values between sets. In Configuration 1 items $1-8$ formed Set 1 , items 9-18 formed Set 2, and items 19-30 formed Set 3. In Configuration 2 items $1-4$ formed Set 1 , items 5-17 formed Set 2, and items 18-30 formed Set 3. In Configuration 3 items 1-5 formed Set 1 and items 6-30 formed Set 2 . Table 5 presents the results of these tests. The Between means were based on all item pairs across item sets.

The pattern of results for $\bar{Q}_{1}$ does not show significantly higher values within item sets than between sets, and the results for $\bar{Q}_{1}$ do not follow the pattern of results for the other fit statistics. Comparing the results for $Q_{2}$ and signed $Q_{2}$ for Configuration 1 , it is apparent that there were both positive and negative local dependence within the item sets; the level of $Q_{2}$ and $Q_{3}$ values were within the range found for the unidimensional simulations.

\section{Table 5}

Mean $\bar{Q}_{1}, Q_{2}$, signed $Q_{2}$, and $Q_{3}$ Values and

Kruskal-Wallis (K-W) Statistics for Item Configurations

for Two-Dimensional Simulated Data

\begin{tabular}{|c|c|c|c|c|c|c|}
\hline $\begin{array}{l}\text { Configu- } \\
\text { ration }\end{array}$ & $\begin{array}{l}\text { Item } \\
\text { set }\end{array}$ & $\begin{array}{l}\text { Number } \\
\text { of Items }\end{array}$ & $\overline{\mathbf{Q}}_{1}$ & $\mathrm{Q}_{2}$ & $\begin{array}{c}\text { Signed } \\
\mathrm{Q}_{2}\end{array}$ & $\mathrm{e}_{3}$ \\
\hline 1 & $\begin{array}{c}1 \\
2 \\
3 \\
\text { Between } \\
K-W\end{array}$ & $\begin{array}{r}8 \\
10 \\
12\end{array}$ & $\begin{array}{l}11 \\
12 \\
16 \\
13 \\
<1\end{array}$ & $\begin{array}{r}10 \\
9 \\
10 \\
10 \\
2\end{array}$ & $\begin{array}{l}-5 \\
-3 \\
-7 \\
-6 \\
<1\end{array}$ & $\begin{array}{r}-.02 \\
-.02 \\
-.03 \\
-.03 \\
<1\end{array}$ \\
\hline 2 & $\begin{array}{c}1 \\
2 \\
3 \\
\text { Between } \\
K-W\end{array}$ & $\begin{array}{r}4 \\
13 \\
13\end{array}$ & $\begin{array}{r}11 \\
11 \\
8 \\
10 \\
<1\end{array}$ & $\begin{array}{l}24 \\
17 \\
15 \\
19 \\
12\end{array}$ & $\begin{array}{r}-24 \\
15 \\
3 \\
-15 \\
139\end{array}$ & $\begin{array}{r}-.08 \\
.04 \\
.00 \\
-.06 \\
155\end{array}$ \\
\hline 3 & $\begin{array}{c}\frac{1}{2} \\
\text { Between } \\
K-W\end{array}$ & $\begin{array}{r}5 \\
25\end{array}$ & $\begin{array}{l}6 \\
8 \\
7 \\
7\end{array}$ & $\begin{array}{l}13 \\
11 \\
11 \\
<1\end{array}$ & $\begin{array}{r}13 \\
-7 \\
-8 \\
4\end{array}$ & $\begin{array}{r}.03 \\
-.03 \\
-.03 \\
3\end{array}$ \\
\hline
\end{tabular}


In Configuration 2 all the local dependence within Item Set 1 was negative, and almost all the local dependence within Item Set 2 was positive; Item Set 3 contained mixed positive and negative local dependence. The within-set and between-set levels of local dependence differed significantly $(p<.001)$ for $Q_{2}$, signed $Q_{2}$, and $Q_{3}$.

In Configuration 3 all the local dependence within Item Set 1 was positive, and almost all the local dependence within Item Set 2 and between sets was negative. The significance probabilities for the Kruskal-Wallis tests were .04 for the signed $Q_{2}$ values and .08 for the $Q_{3}$ values.

\section{Real Data}

Fit measures. The correlations of $\bar{Q}_{1}$ with $Q_{2}$ ranged from .01 to .02 , and the correlations of $\bar{Q}_{1}$ with $Q_{3}$ ranged from -.05 to .03 . There was essentially no relationship between $\bar{Q}_{1}$ and $Q_{2}$ or $Q_{3}$. The correlation between signed $Q_{2}$ and $Q_{3}$ ranged from .78 to .93 , and these two statistics appeared to measure very similar properties of the item pairs.

Significance tests were conducted to determine if the fit statistics followed their null distributions. The probability that the $Q_{1}$ values came from a chisquare distribution with 7 degrees of freedom was $<.005$ for every real data set. The probability that the $Q_{2}$ values came from a chi-square distribution with 10 degrees of freedom was $<.001$ for every real data set, and the probability that the $z$ transformations of the $Q_{3}$ statistics came from a normal distribution with mean zero and variance $1 /(N-3)$ was $<.001$ for every real data set. There were more large $Q_{1}$ and $Q_{2}$ values than expected by their null distributions, and the $z$ statistics tended to be more extreme than expected.

Tables 6 and 7 contain mean values of $\bar{Q}_{1}, Q_{2}$, signed $Q_{2}$, and $Q_{3}$ for the Data Sets in Grade 3 (Table 6) and Grade 6 (Table 7). The Between means were based on all item pairs across item sets. Also in Tables 6 and 7 are the Kruskal-Wallis statistics testing that the within-set fit values arose from the same population as the between-set fit values. Kruskal-Wallis values greater than 6.6 are significant at the .01 level and values greater than 10.8 are significant at the .001 level.
It is clear from Tables 6 and 7 that $\bar{Q}_{1}$ had very different patterns of results from the other fit measures. Item sets that had high $\bar{Q}_{1}$ values did not necessarily have high $Q_{2}$ or $Q_{3}$ values. In other words, item pairs that appeared locally dependent using $Q_{2}$, signed $Q_{2}$, or $Q_{3}$ did not individually tend to have poor fit between model predictions and observations of item characteristic curves.

The signed and unsigned $Q_{2}$ values can be compared to reveal how much local dependence there is and its direction. The change in magnitude from the unsigned to the signed $Q_{2}$ reflects how much of the local dependence is negative. The signed $Q_{2}$ values and the $Q_{3}$ values are more useful than unsigned $Q_{2}$ values for comparing the within-set and between-set local dependence, because the between-set unsigned $Q_{2}$ values can be large due to negative local dependence. Almost every withinset mean signed $Q_{2}$ value and mean $Q_{3}$ value was positive and every between-set mean signed $Q_{2}$ value and mean $Q_{3}$ value was negative, indicating that the item sets did tend to define different traits. Every signed $Q_{2}$ Kruskal-Wallis test and every $Q_{3}$ Kruskal-Wallis test was significant at less than the .01 level except for Data Sets 1 and 6.

For Data Set 2, which is based on Grade 3, Dividing items were highly locally dependent. However, by Grade 6 the same type of item grouping (Data Set 10) did not show a high degree of local dependence for the Dividing items. Data Set 3 showed a great deal of local dependence within item sets such as Adding with regrouping, Subtracting with regrouping, Multiplying by 5 , Dividing with $\div$ notation, and Dividing with $\sqrt{ }$ notation. For Data Set 11 Fractions computations were locally dependent, and Data Set 12 showed that Adding decimals, Adding fractions, and Subtracting fractions had particularly high local dependence.

For Data Sets 5 and 14 there was not an extremely high degree of local dependence in the a priori category objectives except for Sequences (Grades 3 and 6) and Number theory (Grade 6 only). However, by examining the $Q_{2}$ values and regrouping the items into Data Sets 6 and 15, some very highly locally dependent item sets were iden- 
Table 6

Mean $\bar{Q}_{1}, Q_{2}$, Signed $Q_{2}$, and $Q_{3}$ Values and Kruskal-Wallis (K-W) Statistics for Data Sets for Grade 3

\begin{tabular}{|c|c|c|c|c|c|c|c|c|c|}
\hline \multirow{2}{*}{$\begin{array}{l}\text { Data Set \& } \\
\text { Item Type }\end{array}$} & \multirow[b]{2}{*}{$\overline{\mathrm{Q}}_{1}$} & \multicolumn{3}{|c|}{ Signed } & \multirow{2}{*}{$\begin{array}{l}\text { Data Set \& } \\
\text { Item Type }\end{array}$} & \multirow[b]{2}{*}{$\overline{\mathrm{Q}}_{1}$} & \multicolumn{3}{|c|}{ Signed } \\
\hline & & $Q_{2}$ & $Q_{2}$ & $Q_{3}$ & & & $Q_{2}$ & $\mathrm{Q}_{2}$ & $Q_{3}$ \\
\hline Data set 1 & & & & & Data Set 6 & & & & \\
\hline CTBS/U & 28 & 27 & 1 & -.01 & Graph read & 10 & 124 & 124 & .26 \\
\hline DMI & 16 & 30 & 14 & .02 & Clock & 34 & 78 & 78 & .19 \\
\hline Between & 22 & 16 & -7 & -.03 & Nearest ten & 17 & 54 & 54 & .09 \\
\hline $\mathrm{K}-\mathrm{W}$ & 53 & 23 & $<1$ & 2 & Place value & 20 & 208 & 208 & .28 \\
\hline Data set 2 & & & & & Number sent & 14 & 60 & 60 & .13 \\
\hline Add & 25 & 33 & 24 & .04 & Even numbers & 25 & 146 & 146 & .22 \\
\hline Subtract & 22 & 29 & 17 & .03 & Before & 14 & 240 & 240 & .36 \\
\hline Multiply & 31 & 35 & 32 & .08 & Number 1 ine & 23 & 91 & 91 & .18 \\
\hline Divide & 18 & 175 & 175 & .29 & Multiply & 19 & 71 & 71 & .15 \\
\hline Between & 25 & 17 & -12 & -.04 & Fractions & 14 & 639 & 639 & .55 \\
\hline $\mathrm{K}-\mathrm{W}$ & $<1$ & 23 & 285 & 309 & Sequences & 6 & 155 & 155 & .25 \\
\hline Data set 3 & & & & & Shapes & 13 & 76 & 76 & .17 \\
\hline Add $w / 0 \mathrm{rg}$ & 23 & $2 I$ & 20 & .03 & Other & 19 & 11 & -2 & -.01 \\
\hline Add with rg & 26 & 78 & 73 & .14 & Between & 19 & 11 & -3 & -.01 \\
\hline Sub w/o rg & 28 & 26 & 23 & .05 & $\mathrm{~K}-\mathrm{W}$ & 17 & $<1$ & 8 & 6 \\
\hline Sub with rg & 15 & 76 & 76 & .15 & Data Set 7 & & & & \\
\hline Mult by 5 & 25 & 50 & 50 & .13 & CTBS $/ \mathrm{U}$ & 24 & 26 & 11 & .01 \\
\hline Other mult & 34 & 28 & 24 & .07 & DMI & 17 & 13 & 4 & .00 \\
\hline Divide $\div$ & 27 & 207 & 207 & .32 & Between & 20 & 12 & -7 & -.03 \\
\hline Divide $\sqrt{ }$ & 8 & 206 & 205 & .31 & $\mathrm{~K}-\mathrm{W}$ & 1 & 3 & 227 & 334 \\
\hline Between & 24 & 19 & -9 & -.03 & Data set 8 & & & & \\
\hline $\mathrm{K}-\mathrm{W}$ & 2 & 71 & 249 & 249 & CTBS/U & 20 & 12 & -2 & -.01 \\
\hline Data set 4 & & & & & DMI & 20 & 43 & 39 & .07 \\
\hline CTBS/U & 19 & 11 & 0 & .00 & Between & 20 & 12 & -7 & -.03 \\
\hline DMI & 19 & 12 & 5 & .01 & $\mathrm{~K}-\mathrm{W}$ & $<1$ & $<1$ & 75 & 118 \\
\hline Between & 19 & 11 & -5 & -.02 & & & & & \\
\hline $\mathrm{K}-\mathrm{W}$ & $<1$ & $<1$ & 250 & 460 & & & & & \\
\hline Data set 5 & & & & & & & & & \\
\hline Count \& match & 12 & 23 & 21 & .03 & & & & & \\
\hline Numeration & 19 & 16 & 4 & .00 & & & & & \\
\hline Number theory & 23 & 14 & 5 & .00 & & & & & \\
\hline Measurement & 22 & 12 & 1 & .00 & & & & & \\
\hline Geometry & 18 & 19 & 16 & .04 & & & & & \\
\hline Problem solv & 18 & 13 & 4 & .00 & & & & & \\
\hline Number sent & 19 & 19 & 11 & .01 & & & & & \\
\hline Sequences & 9 & 59 & 59 & .10 & & & & & \\
\hline Between & 19 & 11 & -3 & -.01 & & & & & \\
\hline$K-W$ & 6 & $<1$ & 62 & 59 & & & & & \\
\hline
\end{tabular}

tified. Although all the item pairs with similar content were not necessarily locally dependent, all the item pairs with high $Q_{2}$ or $Q_{3}$ values had clear content similarities. It should be noted that the Kruskal-Wallis tests are not helpful with Data Sets 6 and 15 because of the Other group. The Other group, while classified as an item set, was actually a collection of items that did not have noticeably high $Q_{2}$ values. Thus, when the Kruskal-Wallis test was performed, the Other $Q_{2}$ or $Q_{3}$ values were very similar to the Between $Q_{2}$ or $Q_{3}$ values. Also, because the $Q_{2}$ values were used in the classification of items into sets for Data Sets 6 and 15, the $Q_{2}$ values had to be higher for those sets. The mean $Q_{2}$ and $Q_{3}$ values for Data Sets 6 and 15 are of interest, however, because they show the amount 
Table 7

Mean $\bar{Q}_{1}, Q_{2}$, signed $Q_{2}$, and $Q_{3}$ Values and Kruskal-Wallis $(K-W)$

Statistics for Data Sets for Grade 6

\begin{tabular}{|c|c|c|c|c|c|c|c|c|c|}
\hline $\begin{array}{l}\text { Data Set \& } \\
\text { Item Type }\end{array}$ & $\bar{Q}_{1}$ & $Q_{2}$ & $\begin{array}{c}\text { Signe } \\
\mathrm{Q}_{2}\end{array}$ & $Q_{3}$ & $\begin{array}{l}\text { Data Set \& } \\
\text { Item Type }\end{array}$ & $\overline{\mathrm{Q}}_{1}$ & $Q_{2}$ & $\begin{array}{c}\text { Signe } \\
\mathrm{Q}_{2}\end{array}$ & $Q_{3}$ \\
\hline Data set 9 & & & & & Data set 14 & & & & \\
\hline CTBS/U & 19 & 17 & 7 & .01 & Number sent & 19 & 26 & 18 & .03 \\
\hline DMI & 24 & 21 & 6 & .00 & Measurement & 18 & 12 & 3 & .01 \\
\hline Between & 21 & 13 & -6 & -.03 & Geometry & 16 & 12 & 7 & .02 \\
\hline $\mathrm{K}-\mathrm{W}$ & 1 & 29 & 215 & 334 & Problem solv & 20 & 11 & 3 & .01 \\
\hline Data set 10 & & & & & Numeration & 15 & 19 & 8 & .00 \\
\hline Add & 17 & 35 & 20 & .02 & Number theory & 21 & 87 & 77 & .06 \\
\hline Subtract & 20 & 20 & 8 & .01 & Sequences & 22 & 134 & 134 & .27 \\
\hline Multiply & 25 & 16 & 4 & .01 & Ineq/Odd/Mult & 17 & 9 & 9 & .01 \\
\hline Divide & 23 & 18 & 10 & .02 & Between & 18 & 11 & -3 & -.01 \\
\hline Between & 21 & 14 & -3 & -.02 & $\mathrm{~K}-\mathrm{W}$ & 1 & 7 & 141 & 198 \\
\hline $\mathrm{K}-\mathrm{W}$ & $<1$ & 14 & 117 & 173 & Data Set 15 & & & & \\
\hline Data set 11 & & & & & Greater than & 10 & 89 & 89 & .15 \\
\hline Whole numbers & 19 & 15 & 5 & .01 & Fractions & 25 & 87 & 83 & .15 \\
\hline Decimals & 22 & 24 & 11 & .01 & Estimation & 20 & 591 & 591 & .52 \\
\hline Fractions & 25 & 48 & 39 & .05 & Seq/Ineq/Odd & 21 & 60 & 58 & .13 \\
\hline Between & 22 & 13 & -6 & -.02 & Conven Msrmt & 11 & 86 & 86 & .19 \\
\hline $\mathrm{K}-\mathrm{W}$ & 4 & 28 & 301 & 297 & Place val DMI & 10 & 784 & 784 & .59 \\
\hline Data set 12 & & & & & Expand notat & 29 & 380 & 380 & .49 \\
\hline Add whole & 18 & 10 & 10 & .04 & Place val CTBS & 11 & 64 & 64 & .14 \\
\hline Sub whole & 21 & 28 & 27 & .06 & Other & 18 & 11 & 0 & -.00 \\
\hline Mult whole & 20 & 21 & 13 & .03 & Between & 19 & 12 & -4 & -.02 \\
\hline Divide whole & 20 & 19 & 16 & .04 & $K-W$ & 20 & 21 & 180 & 290 \\
\hline Add decimals & 16 & 62 & 57 & .08 & Data set 16 & & & & \\
\hline Sub decimals & 23 & 32 & 24 & .04 & СTBS/U & 20 & 19 & 14 & .03 \\
\hline Mult decimals & 26 & 25 & 8 & .01 & DMI & 18 & 15 & 7 & .01 \\
\hline Div decimals & 35 & 37 & 29 & .05 & Between & 19 & 13 & -9 & -.03 \\
\hline Add fractions & 18 & 106 & 106 & .16 & $\mathrm{~K}-\mathrm{W}$ & 1 & 32 & 1176 & 1668 \\
\hline Sub fractions & 21 & 49 & 42 & .06 & Data set 17 & & & & \\
\hline Mult\&Div frac & 44 & 11 & 8 & .02 & CTBS/U & 16 & 13 & 8 & .02 \\
\hline Between & 23 & 14 & -3 & -.02 & DMI & 23 & 21 & 11 & .01 \\
\hline $\mathrm{K}-\mathrm{W}$ & 5 & 47 & 256 & 305 & Between & 19 & 12 & -9 & -.04 \\
\hline Data set 13 & & & & & $\mathrm{~K}-\mathrm{W}$ & $<1$ & 6 & 1110 & 1459 \\
\hline CTBS/U & 15 & 11 & 4 & .01 & & & & & \\
\hline DMI & 20 & 15 & 4 & .00 & & & & & \\
\hline Between & 18 & 11 & -5 & -.02 & & & & & \\
\hline $\mathrm{K}-\mathrm{W}$ & 10 & $<1$ & 382 & 557 & & & & & \\
\hline
\end{tabular}

of local dependence that was evident for some item sets.

When a MC test and a MC\&A test were analyzed together, there was evidence of substantial local dependence (as measured by signed $Q_{2}$ and $Q_{3}$ ), particularly for Grade 6 (see Data Sets 7, 8, 16, and 17). The Grade 6 mixed-content Kruskal-Wallis tests for signed $Q_{2}$ and $Q_{3}$ were the most significant tests of all those computed.

The $Q_{2}$ and $Q_{3}$ values were examined for items at the ends of tests. There was no evidence of local dependence among these items due to their position in the test.

Fit measures and item parameters. In order to determine whether local dependence appeared greater for items with similar item parameters, correlations were obtained between the absolute value of the difference between parameter values for each pair of items and signed $Q_{2}$ or $Q_{3}$. For signed $Q_{2}$ this correlation ranged from -.05 to -.19 for the $\hat{a}_{i}$ 
parameters, from .00 to -.18 for the $\hat{b}_{i}$ parameters, and from -.03 to -.28 for the $\hat{c}_{i}$ parameters. For $Q_{3}$ this correlation ranged from -.05 to -.20 for the $\hat{a}_{i}$ parameters, from .06 to -.11 for the $\hat{b}_{i}$ parameters, and from -.02 to -.40 for the $\hat{c}_{1}$ parameters.

Figure 1 shows the plot of the signed $Q_{2}$ values and the absolute differences between $\hat{b}_{i}$ parameters for Grade 6 CTBS/U-MC DMI-MC items. This plot (which represents a correlation of -.14 ) is typical of the plots for the other tests and parameters for signed $Q_{2}$. Figure 2 is the plot of the $Q_{3}$ values and the absolute differences between $\hat{b}_{i}$ parameters for the same items as those in Figure 1. As seen in a comparison of Figures 1 and 2, the signed $Q_{2}$ values tend to have a tighter plot than the $Q_{3}$ values; in both figures, however, high $Q_{2}$ or $Q_{3}$ values occurred only for items with similar parameters, but most item pairs that had similar parameters did not have high $Q_{2}$ or $Q_{3}$ values.

An analysis was done to see if the item sets that appeared to be locally dependent in Tables 6 and 7 had distinctive parameters. The items were sorted into sets as in Tables 6 and 7 and a Kruskal-Wallis test was used to test the hypothesis that the param- eters for the items in the different sets all came from the same population. Tables 8 and 9 display the means of the parameters by sets and the values of the Kruskal-Wallis statistics. In many cases, but not all, item sets that showed substantial local dependence also showed $\hat{a}_{i}$ or $\hat{b}_{i}$ parameters that differed from items in other sets. For MC the most highly locally dependent item sets tended to be among the most difficult and most discriminating (see Data Sets 2, 3, 11, and 12). For MC\&A the most highly locally dependent item sets were not necessarily extreme in terms of their difficulties or discriminations (see Data Sets 6, 14, and 15). The $\hat{c}_{i}$ parameters did not tend to show significant differences among item sets except for the Grade 6 tests that differed substantially in their numbers of answer choices (see Data Sets 9, 13, 16, and 17).

Trait estimates. As described in the Method section, the Grade 3 MC\&A tests and the Grade 6 $\mathrm{MC}$ tests were divided into sets of items to obtain trait estimates and to examine how local dependence affects equatings. Table 10 displays the mean $\hat{a}_{i}$ and $\hat{b}_{t}$ values and the mean proportion correct for the items entering into each trait estimate. For the Grade 3 tests the CTBS/U items tended to be

\section{Figure 1}

Absolute Difference Between $\hat{b}$, Values for Each Item Pair

Plotted Against Signed $Q_{2}$ for Grade 6 CTBS/U-MC DMI-MC Items

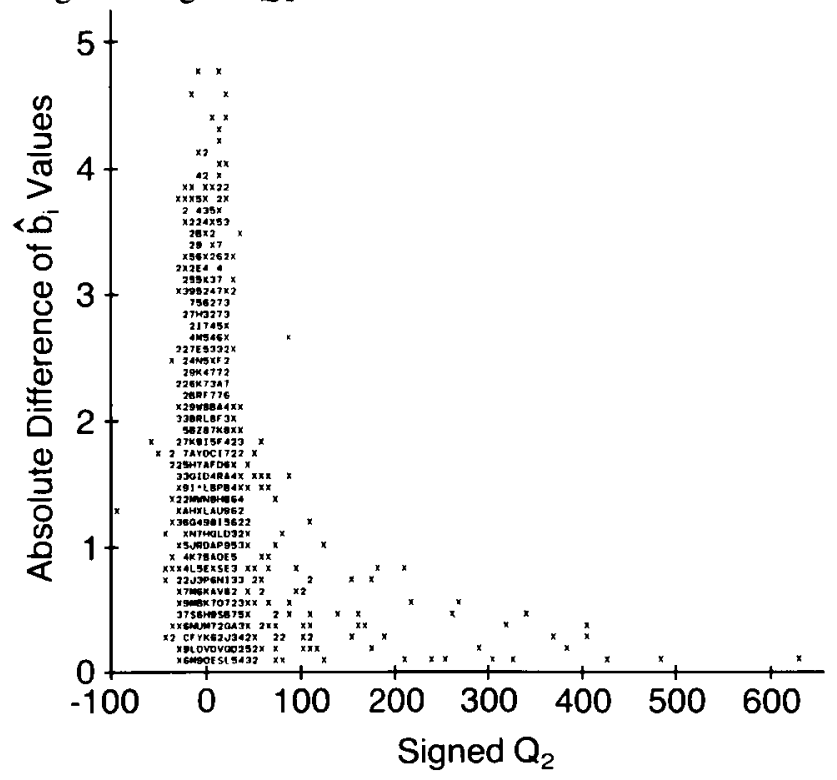


Figure 2

Absolute Difference Between $\hat{b}_{i}$ Values for Each Item Pair

Plotted Against $Q_{3}$ for Grade 6 CTBS/U-MC DMI-MC Items

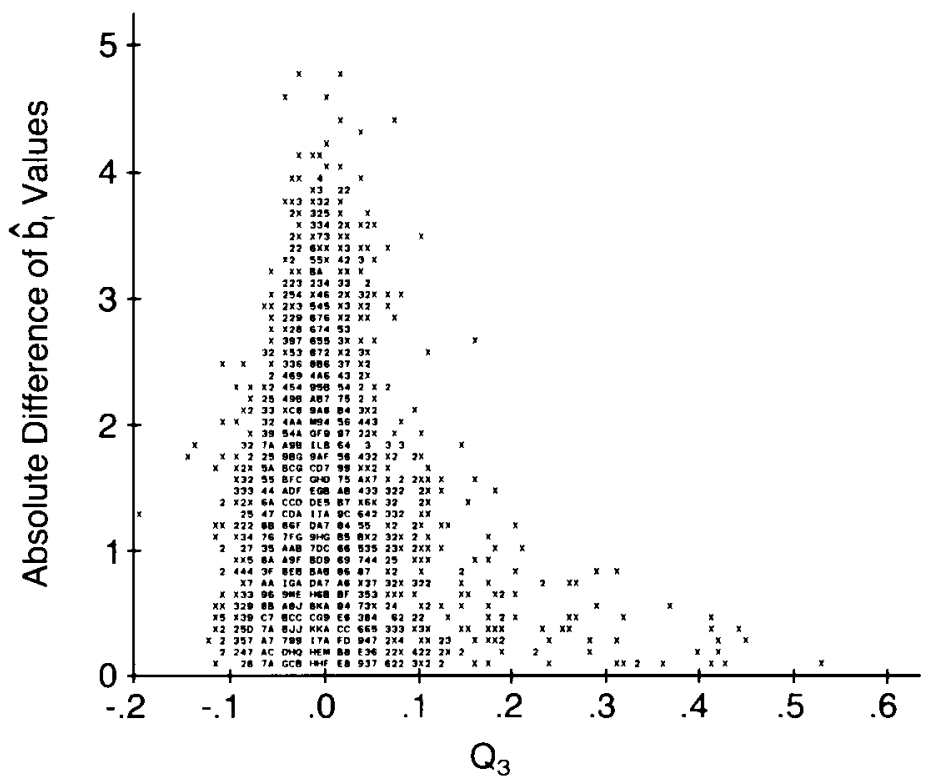

more discriminating and difficult than the DMI items. The Locally Dependent items tended to be slightly more difficult than the Control items. For Grade 6 the CTBS/U and DMI items had similar difficulties and discriminations. Whole number computations tended to be easier than decimal computations, which tended to be easier than fractions computations. The difficulties of the Control items roughly followed the difficulties of the Whole Numbers, Decimals, and Fractions items. Although the Whole Number and Fractions sets (and the Control 1 and Control 3 sets) differed enough in difficulty to be considered to involve vertical equating, all the remaining equatings were horizontal equatings.

The results of the equatings are in Table 11. Compared to the equatings involving the Control tests at Grade 3, the CTBS/U and DMI equating showed a slightly higher standardized mean difference and a substantially lower correlation, higher SRMSD, and greater amount of local bias. The CTBS/U scores tended to be higher than the DMI scores among low scoring examinees and lower than the DMI scores among high scoring examinees. The equatings involving the Locally Dependent item set had the same standardized mean difference as the Control 1 to Control 2 equating but lower correlations and higher SRMSD values.

In Grade 6 the CTBS/U to DMI equating had about the same size standardized mean difference as the other equatings, except for the equatings involving Fractions. The correlation between CTBS/ $\mathrm{U}$ scores and DMI scores was lower than those involving the Control items but higher than those involving the Fractions items. The Whole Numbers and Decimals equating had the same standardized mean difference but a lower correlation than the Control 1 to Control 2 equating. The Fractions equatings had higher standardized mean differences, lower correlations, and higher local bias than the Control 1 to Control 3 equating. An examination of plots of Fractions scores with other scores revealed that examinees who got very low scores on the Fractions items could get fairly high scores on the Whole Numbers and Decimals items. 
Table 8

Mean $\hat{a}_{i}, \hat{b}_{i}$, and $\hat{c}_{i}$ Values and Kruskal-Wallis (K-W) Statistics for Data Sets for Grade 3



Note. The degrees of freedom for the Kruskal-ivallis statistics appear in parentheses following " $\mathrm{K}-\mathrm{W}$ ".

$a_{p<.01}$

\section{Discussion}

\section{Simulated Data}

Properties of the fit measures. $\bar{Q}_{1}$ values had low correlations with $Q_{2}$ and $Q_{3}$ values, and the factors that cause misfit as measured by $\bar{Q}_{1}$ do not appear to include multidimensionality. Van den Wollenberg (1982) found a Rasch fit measure analogous to $Q_{1}$ not to be sensitive to multidimensionality, and Yen (1981) noted that $Q_{1}$ was not useful for determining when a two-parameter model was inappropriately applied to three-parameter data. 
Table 9

Mean $\hat{a}_{i}, \hat{b}_{i}$, and $\hat{c}_{i}$ Values and Kruskal-Wallis (K-W) Statistics for Data Sets for Grade 6

\begin{tabular}{|c|c|c|c|c|c|c|c|}
\hline $\begin{array}{l}\text { Data Set \& } \\
\text { Item Type }\end{array}$ & $\hat{a}_{i}$ & $\hat{b}_{i}$ & $\hat{c}_{i}$ & $\begin{array}{l}\text { Data Set } \& \\
\text { Item Type }\end{array}$ & $\hat{a}_{i}$ & $\hat{b}_{i}$ & $\hat{c}_{i}$ \\
\hline Data set 9 & & & & Data set 14 & & & \\
\hline CTBS/U & .96 & -.32 & .16 & Number sent & .95 & -.09 & .13 \\
\hline DMI & .87 & -.31 & .06 & Measurement & .86 & -.22 & .15 \\
\hline $\mathrm{K}-\mathrm{W} \quad(1)$ & 3 & $<1$ & $48^{a}$ & Geometry & .77 & 1.05 & .13 \\
\hline Data set 10 & & & & Problem solv & .95 & -.06 & .15 \\
\hline Add & 1.00 & -.33 & .12 & Numeration & .96 & -.34 & .17 \\
\hline Subtract & .99 & -.55 & .11 & Number theory & 1.09 & .05 & .13 \\
\hline Multiply & .68 & -.33 & .10 & Sequences & .86 & -1.17 & .03 \\
\hline Divide & 1.01 & -.04 & .10 & Ineg/Odd/Mult & .77 & -.28 & .08 \\
\hline$K-W \quad(3)$ & $13^{a}$ & 4 & $<1$ & $\mathrm{~K}-\mathrm{W}(7)$ & 8 & 18 & 8 \\
\hline Data set 11 & & & & Data set 15 & & & \\
\hline Whole num & .90 & -.75 & .11 & Greater than & 1.27 & .73 & .22 \\
\hline Decimals & .84 & -.26 & .10 & Fractions & .86 & -.04 & .07 \\
\hline Fractions & 1.13 & .55 & .13 & Estimation & 1.42 & .45 & .10 \\
\hline$K-W \quad(2)$ & 2 & $15^{\mathrm{a}}$ & 3 & Seq/Ineq/odd & .84 & -.81 & .05 \\
\hline Data set 12 & & & & Conven Msrmnt & .95 & 1.01 & .09 \\
\hline Add whole & .47 & -1.44 & .11 & Place val DMI & .59 & .54 & .08 \\
\hline Sub whole & .76 & -1.20 & .09 & Expand notat & 1.09 & -.81 & .07 \\
\hline Mult whole & .76 & -1.01 & .10 & Place vl CTBS & .88 & -.69 & .13 \\
\hline Divide whole & 1.12 & -.27 & .11 & Other & .89 & .04 & .16 \\
\hline Add decimal & .96 & -.48 & .12 & $\mathrm{~K}-\mathrm{W} \quad(8)$ & 16 & 12 & 18 \\
\hline Sub decimal & .87 & -.78 & .10 & Data set 16 & & & \\
\hline Mult decimal & .68 & .20 & .10 & CTBS/U & .85 & -.37 & .14 \\
\hline Div decimal & .75 & .50 & .08 & $\mathrm{DMI}$ & .90 & .30 & .10 \\
\hline Add fraction & 1.34 & .54 & .13 & $\mathrm{~K}-\mathrm{W}$ (1) & $<1$ & $9^{a}$ & $10^{\circ}$ \\
\hline Sub fraction & 1.41 & .49 & .14 & Data set 17 & & & \\
\hline Mult\&Div fra & .39 & .65 & .10 & CTBS/U & .93 & -.21 & .25 \\
\hline$K-W \quad(10)$ & $39^{\mathrm{a}}$ & $30^{\mathrm{a}}$ & 6 & DMI & .83 & -.33 & .05 \\
\hline Data set 13 & & & & $\mathrm{~K}-\mathrm{W}$ (1) & 5 & $<1$ & $69^{a}$ \\
\hline $\mathrm{CTBS} / \mathrm{U}$ & .94 & -.28 & .21 & & & & \\
\hline DMI & .89 & .26 & .08 & & & & \\
\hline $\mathrm{K}-\mathrm{W} \quad(1)$ & $<1$ & $7^{\mathrm{a}}$ & $58^{\mathrm{a}}$ & & & & \\
\hline
\end{tabular}

Note. The degrees of freedom for the Kruskal-Wallis statistics appear in parentheses following " $\mathrm{K}-\mathrm{W}$ ". $a_{p<.01}$.

Thus, although $Q_{1}$ can be useful in identifying items that have unexpected item characteristic curves, $Q_{1}$ cannot be relied upon as a complete fit measure.

Signed $Q_{2}$ and $Q_{3}$ produced essentially the same results. It is advantageous to have both signed and unsigned statistics that can be compared, as with signed $Q_{2}$ and $Q_{2}$; these statistics also have magnitudes that are easy to analyze. The small values of the $Q_{3}$ statistics make them more difficult to evaluate subjectively, but the $Q_{3}$ values are readily interpreted as correlations. The $Q_{2}$ values will increase as a function of sample size, while the $Q_{3}$ values will not.

In order to understand the differences in the results for the three two-dimensional simulations, it is necessary to consider the signed $Q_{2}$ or $Q_{3}$ values 
Table 10

Mean Item Difficulties $\left(p_{i}\right), \hat{a}_{i}$, and $\hat{b}_{i}$ Values

for Items Entering Into Trait Estimates

\begin{tabular}{|c|c|c|c|c|c|c|}
\hline Grade & Content & Trait & $\hat{a}_{i}$ & $\hat{\mathrm{b}}_{\mathrm{i}}$ & $\mathrm{P}_{i}$ & $\begin{array}{l}\text { No. of } \\
\text { Items }\end{array}$ \\
\hline \multirow[t]{6}{*}{3} & $M C \& A$ & & & & & \\
\hline & & CTBS/U & 1.09 & -.27 & .63 & 40 \\
\hline & & DMIa & .71 & -.76 & .68 & 40 \\
\hline & & Loc.Dep. & .91 & -.23 & .59 & 27 \\
\hline & & Control 1 & .91 & -.65 & .66 & 26 \\
\hline & & Control 2 & .94 & -.66 & .66 & 25 \\
\hline \multirow[t]{9}{*}{6} & MC & & & & & \\
\hline & & CTBS/U & .96 & -.32 & .62 & 40 \\
\hline & & DMI & .87 & -.31 & .58 & 48 \\
\hline & & Whole num. & .90 & -.75 & .65 & 36 \\
\hline & & Decimals & .84 & -.26 & .57 & 36 \\
\hline & & Fractions & 1.13 & .55 & .41 & 16 \\
\hline & & Control I & .82 & -.57 & .62 & 36 \\
\hline & & Control 2 & 1.04 & .11 & .51 & 36 \\
\hline & & Control 3 & 1.03 & .43 & .47 & 16 \\
\hline
\end{tabular}

Table 11

Comparisons of Trait Estimates

\begin{tabular}{|c|c|c|c|c|c|c|}
\hline$\hat{\theta}_{1}$ & $\hat{\theta}_{2}$ & $\mathbf{r}$ & $\frac{\left(\overline{\hat{\theta}}_{1}-\overline{\hat{\theta}}_{2}\right)}{\bar{S}}$ & $\frac{s_{1}}{s_{2}}$ & SRMSD & $\begin{array}{r}\text { Local } \\
\text { Bias }\end{array}$ \\
\hline \multicolumn{7}{|c|}{ Grade 3 MC\&A } \\
\hline CTBS/U & DIII & .74 & -.05 & .92 & .73 & .07 \\
\hline $\begin{array}{l}\text { Loc.Dep. } \\
\text { Loc.Dep. } \\
\text { Control } 1\end{array}$ & $\begin{array}{ll}\text { Control } & 1 \\
\text { Control } & 2 \\
\text { Control } & 2\end{array}$ & $\begin{array}{l}.81 \\
.80 \\
.85\end{array}$ & $\begin{array}{l}.00 \\
.00 \\
.00\end{array}$ & $\begin{array}{r}1.05 \\
1.01 \\
.96\end{array}$ & $\begin{array}{l}.62 \\
.63 \\
.56\end{array}$ & $\begin{array}{l}.03 \\
.01 \\
.03\end{array}$ \\
\hline \multicolumn{7}{|l|}{ Grade $6 \mathrm{MC}$} \\
\hline $\mathrm{CTBS} / \mathrm{U}$ & DMI & .76 & -.05 & 1.07 & .70 & .06 \\
\hline $\begin{array}{l}\text { Whole num. } \\
\text { Control I }\end{array}$ & $\begin{array}{l}\text { Decimals } \\
\text { Control } 2\end{array}$ & $\begin{array}{l}.77 \\
.89\end{array}$ & $\begin{array}{l}.02 \\
.02\end{array}$ & $\begin{array}{l}.99 \\
.96\end{array}$ & $\begin{array}{l}.68 \\
.48\end{array}$ & $\begin{array}{l}.03 \\
.04\end{array}$ \\
\hline $\begin{array}{l}\text { Whole num. } \\
\text { Decimals } \\
\text { Control } 1\end{array}$ & $\begin{array}{l}\text { Fractions } \\
\text { Fractions } \\
\text { Control } 3\end{array}$ & $\begin{array}{l}.65 \\
.64 \\
.83\end{array}$ & $\begin{array}{l}.09 \\
.08 \\
.04\end{array}$ & $\begin{array}{l}.86 \\
.87 \\
.89\end{array}$ & $\begin{array}{l}.85 \\
.86 \\
.60\end{array}$ & $\begin{array}{l}.17 \\
.16 \\
.09\end{array}$ \\
\hline
\end{tabular}

and to reexamine the simulation conditions in Table 2. In Configuration 1, both underlying traits influenced every item. The correlation between $\hat{a}_{i}$ and the sum of the $a_{i s}$ values used in generating the data was .70. It appears that the unidimensional model used a combination of the two underlying 
traits as the unidimensional trait. When a combination of traits is used to sort examinees into cells, local negative dependence appears. (This effect is described in the earlier section that gives the motivation for the development of signed $Q_{2}$.) Thus, for Configuration 1 , the vast majority of item pairs had negative signed $Q_{2}$ and $Q_{3}$ values. The items did not differ a great deal in terms of their relative $a_{t s}$ values for the two underlying traits, and the within-set and between-set signed $Q_{2}$ and $Q_{3}$ values did not appear significantly different.

For Configuration 2, the correlation between the $\hat{a}_{t}$ values and the $a_{i 1}+a_{t 2}$ values was .89. Again, the unidimensional model used a combination of the underlying traits to define the unidimensional trait. However, in this configuration only the items in Set 1 were influenced by both underlying traits, and these Set 1 items all showed negative local dependence. The items in Sets 2 and 3 were influenced by only one of the underlying traits, and they showed positive local dependence; the between-set local dependence was largely negative.

For Configuration 3, the second trait was a very weak trait compared to the first trait, and the unidimensional trait used to sort examinees into cells was largely Trait 1 . The items in Set 2 , which were influenced only by the first underlying trait, showed a low amount of negative local dependence, as would be consistent with part-whole contamination discussed earlier. The items in Set 1, which were influenced by both underlying traits, showed positive local dependence.

These results for the two-dimensional simulations can be summarized as follows. If a combination of two underlying traits is used as the unidimensional trait, then items that are influenced by both underlying traits will show negative local dependence and items that are influenced by only one underlying trait will show positive local dependence. If only one of the underlying traits is used as the unidimensional trait, then items that are influenced only by that underlying trait will show slight negative local dependence due to part-whole contamination and items that are influenced by both underlying traits will show positive local dependence.

\section{Real Data}

Properties of the fit measures. The real data sets tended to have positive local dependence within sets and negative local dependence between sets. These results appear most consistent with a multidimensional situation in which there are important, different underlying traits influencing the items within the different item sets and the unidimensional estimated trait is a combination of these underlying traits.

The overall significance tests involving $Q_{2}$ or $Q_{3}$ were not particularly useful by themselves. It was important to examine the direction of the local dependence both within and between sets of items that were suspected of being multidimensional. It was also important to keep in mind that signed $Q_{2}$ and $Q_{3}$ will have slightly negative values for unidimensional data, and that any positive correlations that are found may be underestimates of the strength of the positive relationships between the items.

For the real data, $Q_{2}$ and $Q_{3}$ identified item sets as being locally dependent that a priori would appear to be so. For the MC tests the locally dependent item sets corresponded to category objectives that were created through a logical analysis of the item content. For the MC\&A tests the category objectives did appear to be locally dependent, but item sets that were much more locally dependent than the category objectives were identified through an examination of the $Q_{2}$ values. (These same locally dependent item sets would be identified through an examination of $Q_{3}$ values.) Thus, the construct validity of $Q_{2}$ and $Q_{3}$ was enhanced by the fact that every item set that was identified as having high $Q_{2}$ or $Q_{3}$ values had a logical association of content among the items in the set. $Q_{2}$ and $Q_{3}$, therefore, appeared useful for sorting items into sets measuring different dimensions. This information can be valuable in both test construction and in test scoring.

$Q_{2}$ and $Q_{3}$ also distinguished between items that might be expected to be locally dependent. For example, for Data Set 15 CTBS/U place value items and DMI place value items were locally dependent within each set but not across sets. A closer ex- 
amination of the items revealed that while the item stems had the same format, the types of answer choices were quite different for the two tests; this difference in the answer choices also affected the difficulty and discrimination of the items. Thus, $Q_{2}$ or $Q_{3}$ could be useful in item tryouts to distinguish among item formats and to help identify the most useful ones.

Properties of locally dependent items. The items with high $Q_{2}$ or $Q_{3}$ values tended to have similar item parameters, but the items with similar parameters did not necessarily have high $Q_{2}$ or $Q_{3}$ values. High values of $Q_{2}$ or $Q_{3}$ were not artifacts of similarity of item parameters. Similarity of item parameters is a necessary, but not a sufficient condition for high $Q_{2}$ or $Q_{3}$ values. Items need to share one or more unique traits or dimensions in addition to having similar item parameters in order to have high $Q_{2}$ or $Q_{3}$ values. The $Q_{2}$ values are a function of the within-cell $\phi_{i j r}$ values, and the $\phi_{i j r}$ values are restricted in magnitude unless items have similar within-cell $p$ values; an analogous effect occurs with $Q_{3}$. Thus, locally dependent items that do not have similar item parameters are unlikely to have high $Q_{2}$ or $Q_{3}$ values; it is not known how often such items occur.

The MC items that were highly locally dependent tended to have high discriminations and difficulties, but the MC\&A items did not. The MC items tended to be naturally hierarchical in nature. For example, students learn addition without regrouping before they learn addition with regrouping, and they tend to learn some addition before they learn subtraction or multiplication. The MC item sets tend to be correlated and cumulative. For example, to do some division items, examinees need to be able to add, subtract, and multiply. Given this structure of the MC items, it is not surprising that the more complex items appeared to be locally dependent and have high discriminations and difficulties.

The highly locally dependent MC\&A items tended to be "pockets" of separate, fairly independent concepts. Although there would tend to be some correlation among these concepts due to individual differences in speed of progress through the cur- riculum, many of the MC\&A concepts could be learned fairly independently of the others, and these concepts were not strongly cumulative in nature. Unlike the MC items, there is no reason to expect the MC\&A locally dependent items to be highly discriminating or difficult.

Unidimensional trait estimates. It can be hypothesized that when the unidimensional three-parameter model analyzes test data generated from several correlated underlying traits, the unidimensional model uses a combination of the underlying traits as its unidimensional trait. Yen (1984) presented derivations and simulated data that support this hypothesis. McKinley (1983) reported supporting simulation data for the two-parameter model. The fact that local dependence between sets was negative also supports the hypothesis that a combination of traits was used to create the unidimensional trait estimate.

The hypothesis that the unidimensional trait is a combination of correlated underlying traits is consistent with the fact that with the MC data the more complex items tended to have higher discriminations; it is also consistent with the fact that for Grade $3 \mathrm{MC} \& \mathrm{~A}$, the CTBS/U items had higher average discriminations than the DMI items. For the Grade 3 CTBS/U MC\&A test, examinees must read text associated with each item. For the Grade 3 DMI MC\&A items, any associated text is read to the examinees. Thus, it can be hypothesized that the unidimensional model chooses a combination of mathematics and reading skills to define its trait; because the CTBS/U items involve both traits, they tend to have higher discriminations.

It can also be hypothesized that when a test involves independent traits that influence only a few items, such traits are ignored in the definition of the unidimensional three-parameter trait. Reckase (1979) reported results that support this hypothesis. The present study did not include simulations with independent traits, so it does not assist in the evaluation of this hypothesis; but the hypothesis is consistent with the results for the MC\&A tests where the pockets of locally dependent items did not have particularly high discriminations.

Equating tests in the presence of local depen- 
dence. Trait estimates based on items measuring different dimensions had lower correlations than trait estimates that had items that shared dimensions. However, except for the Grade 6 equatings involving the Fractions items, there were not large systematic errors of equating due to the attempted equating of items measuring different dimensions. As might be expected if fractions are frequently taught after whole numbers and decimals, examinees could get moderately high Whole Numbers or Decimals scores while having very low Fractions scores. Thus, it appears that while substantial unsystematic errors of equating are to be expected from the equating of tests involving collections of different dimensions, substantial systematic errors of equating might not be expected unless the two tests measure quite different dimensions that are taught sequentially and that differ in difficulty. Only a handful of trait estimates have been compared here, and a wide variety of conditions can occur with multidimensional tests. It would be prudent to cross-validate any equatings that appear to involve multidimensional tests.

When maximum likelihood scoring of item responses is used with the three-parameter model, item discriminations are used in the item weights. Thus, when a test measures several correlated dimensions, items that measure more of these dimensions will tend, all else being equal, to have higher discriminations and to be given more weight in the test scoring. An alternative scoring procedure is to use unit item weights, so that a number-correct score is converted to a trait estimate (Yen, in press). If the test user wants to measure several dimensions with one score, IRT scaling combined with differential item weighting is a potentially efficient method of test scoring. The desirability of this procedure is a function of whether the additional dimensions are legitimate aspects of the behavior that the test user wants to measure. In any multidimensional testing situation it would be important to review the item discriminations and fit statistics such as $Q_{2}$ or $Q_{3}$ to help determine what dimensions are influencing test performance. Whether or not items are differentially weighted and whether or not IRT is used, when a test measures several dimensions, examinees' scores will be influenced by all these dimensions. Thus, unsystematic and systematic errors of equating might be expected from any scaling and equating procedures that are applied to some multidimensional tests.

\section{References}

CTB/McGraw-Hill. (1981). Comprehensive Tests of Basic Skills, Form U. Monterey CA: Author.

CTB/McGraw-Hill. (1982a). Comprehensive Tests of Basic Skills, Forms $U$ \& V, Preliminary Technical Report. Monterey CA: Author.

CTB/McGraw-Hill. (1982b). Comprehensive Tests of Basic Skills, Forms $U$ \& V, Test Coordinator's Handbook. Monterey CA: Author.

Doody-Bogan, E., \& Yen, W. M. (1983, April). Detecting multidimensionality and examining its effects on vertical equating with the three-parameter logistic model. Paper presented at the meeting of the American Educational Research Association, Montreal.

Gessel, J. (1975a). Diagnostic Mathematics Inventory. Monterey CA: CTB/McGraw-Hill.

Gessel, J. (1975b). Diagnostic Mathematics Inventory Teacher's Guide. Monterey CA: CTB/McGraw-Hill.

Hays, W. L. (1973). Statistics for the social sciences. San Francisco: Holt, Rinehart, \& Winston.

Kingston, N. M., \& Dorans, N. J. (1982). The feasibility of using item response theory as a psychometric model for the GRE Aptitude Test (ETS Research Report 82-12). Princeton NJ: Educational Testing Service.

Lord, F. M. (1953). The relation of test score to the trait underlying the test. Educational and Psychological Measurement, 13, 517-549.

McKinley, R. L. (1983, April). A multidimensional extension of the two-parameter logistic latent trait model. Paper presented at the meeting of the National Council on Measurement in Education, Montreal.

Reckase, M. D. (1979). Unifactor latent trait models applied to multifactor tests: Results and implications. Journal of Educational Statistics, 4, 207-230.

Reckase, M. D., \& McKinley, R. L. (1983, April). The definition of difficulty and discrimination for multidimensional item response theory models. Paper presented at the meeting of the American Educational Research Association, Montreal.

Siegel, S. (1956). Nonparametric statistics for the behavioral sciences. New York: McGraw-Hill.

Traub, R. E. (1983). A priori considerations in choosing an item response model. In R. K. Hambleton (Ed.), 
Applications of Item Response Theory (Monograph). British Columbia: Educational Research Institute of British Columbia.

van den Wollenberg, A. L. (1982), Two new test statistics for the Rasch model. Psychometrika, 47, 123140.

Wingersky, M. S., Barton, M. A., \& Lord, F. M. (1982). LOGIST User's Guide. Princeton NJ: Educational Testing Service.

Yen, W. M. (1981). Using simulation results to choose a latent trait model. Applied Psychological Measurement, 5, 245-262.

Yen, W. M. (1983a). Tau equivalence and equipercentile equating. Psychometrika, 48, 353-370.

Yen, W. M. (1983b). Use of the three-parameter logistic model in the development of a standardized achievement test. In R. K. Hambleton (Ed.), Applications of
Item Response Theory (Monograph). British Columbia: Educational Research Institute of British Columbia.

Yen, W. M. (1984, June). Increasing item complexity: A possible cause of scale shrinkage for unidimensional item response theory. Paper presented at the meeting of the Psychometric Society, Santa Barbara CA.

Yen, W. M. (in press). Obtaining maximum likelihood trait estimates from number-correct scores for the threeparameter logistic model. Journal of Educational Measurement.

\section{Author's Address}

Send requests for reprints or further information to Wendy M. Yen, CTB/McGraw-Hill, 2500 Garden Road, Monterey CA 93940 , U.S.A. 